Article

\title{
Dynamic Tariff for Day-Ahead Congestion Management in Agent-Based LV Distribution Networks
}

\author{
Niyam Haque ${ }^{1, *} \mathbb{D}$, Anuradha Tomar ${ }^{1}\left(\mathbb{D}\right.$, Phuong Nguyen ${ }^{1,2}$ and Guus Pemen ${ }^{1}$ \\ 1 Department of Electrical Engineering, Eindhoven University of Technology, 5600 MB Eindhoven, \\ The Netherlands; a.tomar@tue.nl (A.T.); p.nguyen.hong@tue.nl (P.N.); a.j.m.pemen@tue.nl (G.P.) \\ 2 Sustainable Energy Systems Group, SUSTAIN-ERIN, Luxembourg Institute of Science and Technology, \\ L-4422 Belvaux, Luxembourg \\ * Correspondence: a.n.m.m.haque@tue.nl; Tel.: +31-40-247-8515
}

Received: 1 November 2019; Accepted: 6 January 2020; Published: 9 January 2020

\begin{abstract}
Capacity challenges are becoming more frequent phenomena in residential distribution networks with new forms of loads, distributed renewable energy resources (RES) and price-responsive applications. Different types of demand response programs have been introduced to tackle these challenges through iterative changes in price and/or contractual participations based on incentives. In this research, a dynamic network tariff-based demand response program is proposed to address congestion problems in low-voltage (LV) networks. The formulation takes advantage of the scalable architecture of the agent-based systems that allows local decision making with limited communication. Energy consumption schedules are developed on a day-ahead basis depending on the expected cost of overloading for a number of probable scenarios. The performance of the proposed approach has been tested through simulations in the unbalanced IEEE European LV test feeder. Simulation results reveal up to $82 \%$ reduction in congestion on a monthly basis, while maintaining the quality of supply in the network.
\end{abstract}

Keywords: congestion management; dynamic tariff; agent-based distribution networks; demand response

\section{Introduction}

Fundamental changes are expected in the power systems in terms of increasing shares of renewable energy resources (RES)-based local distributed generation (DG) units and price-responsive residential flexible loads. Due to the intermittent nature of the DG units and reduced diversity of the loads, more and more operational challenges are appearing in residential LV networks in terms of voltage variations, thermal overloading and power quality issues [1]. Congestion or thermal overloading result from power flows through a network asset (lines, cables, transformers) exceeding its transfer capability. Even though the network assets are usually designed to withstand power flows beyond a defined margin, continuous overloading leads to ageing and subsequent failure of the distribution cables and transformer windings [2,3]. The conventional approach of reinforcing the network assets to tackle such issues necessitates a huge investment, and it is also deemed redundant, as the frequency of such issues is unpredictable [4]. In order to avoid the huge costs, different types of demand response (DR) mechanisms have been studied and developed worldwide for the procurement of flexibility from end-users in order to solve these operational challenges. These mechanisms can be roughly subdivided into direct and indirect load control methods. In direct methods, end-users are compensated through an appropriate contract, which enables automatic or manual shift in consumption of electricity based on the requirements of the network operator. Indirect methods, on the other hand, invoke 
flexibility through a market-based coordination and/or dynamic price signals [5,6]. An overview of such mechanisms has been presented in [7-12] for various types of smart grid domains such as, for instance, microgrids, load balancing services, smart electric vehicle (EV) charging and residential energy management.

A centralized coordinated home energy management system, which implements daily power based network tariffs to mitigate network congestion due to the high demands of heat pumps (HPs) and EVs, is described in [13]. In contrast to conventional centralized methods, a number of decentralized approaches have been proposed in order to adequately address the comfort of the end-users for scheduling the energy consumption of dynamic inputs [14-17]. Such decentralized approaches aim to enable the end-users to take autonomous actions with no or limited communication with other entities. Contrary to the centralized approaches, they offer a more robust and reliable operation due to the lower vulnerability for communication failure.

A local market based framework has been presented in [18] that supports the network operators and prosumers to fully utilize the available flexibility and, thus, enhance the operational efficiency of the network. Dynamic tariff schemes that consider the high level penetration of EV and HP have been discussed in [19-21]. In order to tackle congestion in distribution networks, a dynamic subsidy (DS) method is presented in [22] to motivate aggregators to adjust the load profiles and to take network constraints into consideration. DS is inherently an incentive or reward that the distribution system operator (DSO) is willing to pay to the aggregators who would reschedule their energy profiles. Sensitivity analyses and linear approximation methods have been applied in [23] to develop a pricing model, considering EVs as a flexible load and accounting for three-phase and single-phase voltage variation, network losses and overloading of network components. For the identification of barriers, potential solutions and opportunities, a generic method is applied in the Netherlands [24]. The applied method focuses on market integration aspects and tries to access the flexibility procurement mechanism through the aggregators. As an outcome of the study, an analysis on relevant issues for market integration with reference to the Dutch system and European Grid codes is presented and a lack of proper communication system between users is identified. Authors of [25] have performed an analysis to understand the network pricing methodology most suitable for prosumers in the future, and they concluded that contracts would be economically efficient when retailers have access to the full range of devices, which helps them to convert wholesale electricity pricing in retail contracts, and second, the wholesale price associated with customers must represent the short term marginal cost of electricity production.

However, for a market-based DR mechanism, a sound methodology for congestion management is important to reflect the realized ageing of the network assets to a representative financial loss. An integrated congestion management mechanism has been proposed in $[2,16]$ for the residential networks that involve the dynamic loading model of a distribution transformer. In reality, procurement of flexibility can be a more complex problem involving multiple involved actors in the same network. This work extends the market-based control proposed in [16] for day-ahead scheduling of the residential appliances that considers the dynamic network tariff to avoid thermal overloading of the MV/LV transformer. The tool developed in [16] has been used for estimating the overloading cost for different scenarios of imminent day-ahead loading, and a more realistic approach has been adopted of changing the network tariff dynamically for various scenarios of loading. Thus, the new approach becomes robust for uncertainties associated with day-ahead scheduling. The method will take advantages of Multi-Agent Systems (MAS) for a scalable architecture and distributed intelligence in a multi-actor setting. In principle, the method allows for local decision making and dynamic scheduling of the appliances with limited communication and a smaller number of iterations among the actors. Key contributions of this article can thus be listed as follows:

- Day-ahead market-based congestion management through agent-based scheduling of the residential appliances; 
- The estimated incurred cost of overloading is determined through the dynamic loading model of the transformer and used as the trigger for the procurement of flexibility;

- The dynamic network tariff has been calculated through a robust formulation considering uncertainties in loading and correlation among loading in different time steps.

The rest of this paper is organized as follows: Section 2 highlights the overall approach and system architecture; Section 3 presents the problem formulation along with the description of the proposed methodology; Section 4 provides the description of the simulation test case and the assumptions adopted. Finally, simulation results are discussed in Section 5, before summarizing and concluding with Section 6.

\section{Approach}

\subsection{Overview}

With the increasing adoption of flexible domestic appliances and small-scale generation technologies like rooftop solar PV, market-based control of the LV network has been drawing extensive research attention in recent years [26-28]. This has principally paved the way for new market actors (e.g., aggregators, energy service companies), novel business models, innovative control algorithms and in general, a more decentralized operation of the future power system [4].

Transactive energy frameworks have been developed to incorporate the flexibility of residential appliances with a range of scopes and aims $[29,30]$. In this context, market-based mechanisms with local flexibility services have attracted notable interest for ancillary services and network congestion management [6]. However, in a market-coordinated network with a considerable penetration of price-responsive appliances, the overall peak loads tend to be significantly higher due to the higher coincidence of loads in low-price hours. These peak loads may lead to more frequent occurrences of network congestions in the LV networks.

In this work, we aim to incorporate a variable network tariff with the day-ahead dynamic price, as discussed in [6]. The market-based control is coordinated by a commercial market actor such as an aggregator. Aggregators are usually responsible for managing the flexibility in their cluster of end-users and providing the DSO with a schedule of the loading for the following day. Case congestion is expected, and the dynamic price levels are adjusted by varying the network tariff to shift the flexible demand in order to relieve the congestion. Conventional market-based mechanisms try to adjust the day-ahead price based on the network or market issues. These processes need to be carried out by market actors, like aggregators or energy suppliers, who need to be compensated for the provided services and the adjustment in the portfolio. On the contrary, the proposed approach focus on the network tariff part of the price and does not have to depend on the market parties.

The application of such an approach relies on robust and reliable communication among different entities in the whole value chain. An MAS-based system architecture is adopted in this work that provides for an efficient platform for smooth coordination among the involved actors. An overview of the distributed system architecture and related interactions are presented in the following subsection.

\subsection{MAS-Based System Architecture}

A hierarchical MAS-based architecture is adopted in this work (as shown in Figure 1), consisting of device agents, household agents, an aggregator agent and a network agent. The connected loads and DG units are represented by device agents (DAs). The DAs are responsible for the energy optimization of respective appliances based on the given price signals and associated constraints. DAs of each house are coordinated by a house agent (HA) that interfaces with the external market and network agents through an aggregator. The aggregator coordinates the end-users through dynamic prices and supports the DSO with the required flexibility during network issues. 


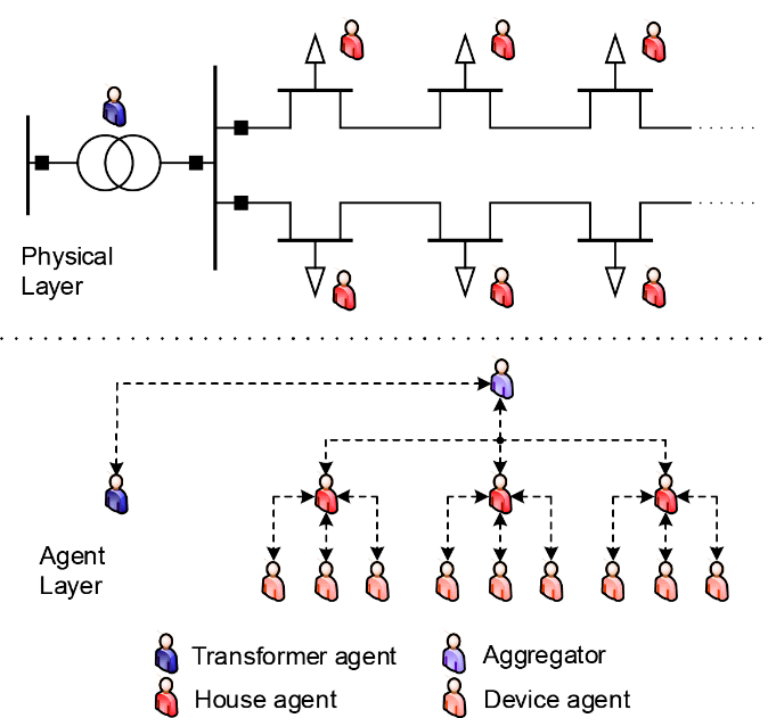

Figure 1. Multi-Agent Systems (MAS)-based system architecture.

The transformer agent (TA) represents a network agent that monitors and controls the MV/LV transformer. The agent-based architecture enables the system to be sufficiently scalable, since more end-users and their appliances can be easily included in the system in terms of new agents. At the same time, the platform can be expanded to wider network areas by implementing more transformers in terms of additional TAs. A more detailed description of the system architecture can be found in [16].

The interactions among the agents in the MAS environment are depicted in Figure 2. In normal operations, the HA adjusts overall energy consumption by optimizing the predicted consumption of the DAs. The final day-ahead profile is sent to the TA to check network security constraints. In case the TA detects an imminent overloading of the transformer, the tariff-based DR phase is triggered (as depicted in Figure 2b). At this phase, the TA calculates the resulting ageing and thermal overloading cost. Based on the cost, it determines required changes in the network tariff at different time instants of the day while keeping the daily average constant. The updated network tariff is sent to the HAs as a flexibility request through the aggregator for an iterative solution of the problem.

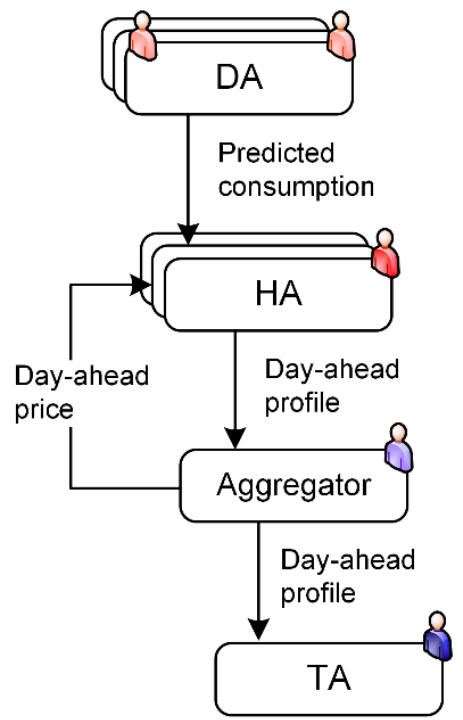

(a) Normal operations

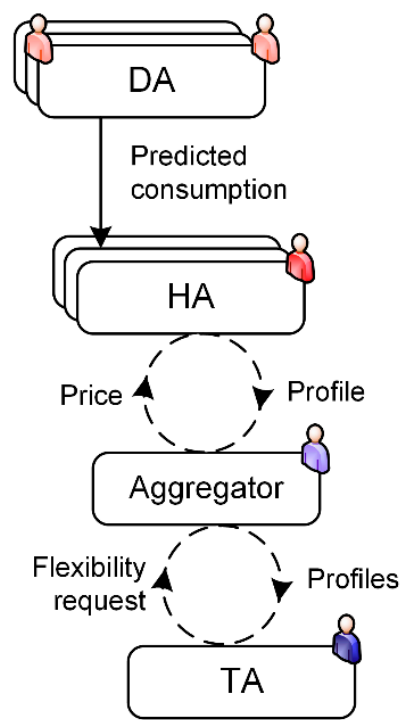

(b) Tariff-based DR

Figure 2. Interaction among the involved agents. 


\section{Modeling}

In this section, the modeling of the proposed mechanism is discussed in detail. At any given time, the total available flexibility depends upon the type of loads in the network. Therefore, in order to procure the required flexibility, the behavior of the loads for different price levels needs to be taken into consideration. The Markov Chain Monte-Carlo method is applied in this regard to perform the modeling of household loads [31].

The day-ahead scheduling of the appliances has two aspects. First, the response of the devices needs to be modeled for dynamic prices, and then their behavior needs to be adjusted for changes in the price if flexibility is requested by the TA.

\subsection{Response to Dynamic Pricing}

The household appliances can be broadly categorized into four groups, i.e., non-controllable, buffer appliances, time shifting and curtailable appliances [32]. The non-controllable appliances are mostly commonly known as the base load and include the devices that do not offer controllability for different levels of price. Buffer appliances represent devices with integrated storage facilities in terms of thermal (e.g., freezers, heat pump) or electro-chemical energy (e.g., batteries, EV). Time-shifting appliances can shift their consumption in time based on price and include the washing machine, dishwasher, etc. Finally, the curtailable appliances refer to the devices that can be interrupted based on predefined contracts for different purposes (e.g., solar PV system for voltage violation or network congestion). Each type of these appliances would react differently to the incoming price levels. The HA tries to optimize the energy cost of the house by utilizing the available flexible loads, and the associated optimization problem could be expressed as:

$$
\min \sum_{a=1}^{|A|} \sum_{t=1}^{N_{T}} p_{t} \times P_{t, a} \times \Delta t
$$

where $A$ is the set of all appliances within a single household, $N_{T}$ is the number of time steps in a day, $p_{t}$ is the price at time $t, P_{t, a}$ is the power of appliance $a$ at time $t$ and $\Delta t$ is the duration of each time step.

The buffer and time shifting appliances need to optimize their profiles based on the dynamic price. Since the devices are categorized in different groups, their energy use can be considered independent of each other. Equation (1) can thus be re-written as:

$$
\sum_{a=1}^{|A|} \min \sum_{t=1}^{N_{T}} p_{t} \times P_{t, a} \times \Delta t .
$$

The behavior of the buffer appliances can be formulated as:

$$
\min \sum_{t}^{N_{T}} p_{t} \times P_{t, a} \times \Delta t \quad \forall a \in A^{b f}
$$

subject to,

$$
\begin{gathered}
\sum_{k+x_{a}}^{t=k} P_{t, a}=\sum_{k+x_{a}}^{t=k} P_{t, a}^{0} \quad \forall a \in A^{b f}, k \in T \\
\min _{T} P_{t, a}^{0}<P_{t, a}<\max _{T} P_{t, a}^{0} \quad \forall a \in A^{b f}, t \in T
\end{gathered}
$$

where $x_{a}$ is the maximum buffer time for appliance $a, P_{t, a}^{0}$ is the original load of appliance $a$ at time $t, T$ is the set of the time steps in a day and $A^{b f}$ is the set of the buffer appliances. The constraints in Equations (4) and (5) control that the total energy consumed by the device will remain constant and buffer time, $x_{a}$, could be altered depending on the price. 
For time shifting devices, the behavior can be represented as the following optimization problem:

$$
\min \sum_{t=1}^{N_{T}} p_{t} \times P_{t+\tau, a} \times \Delta t \quad \forall a \in A^{t s}
$$

subject to,

$$
\begin{gathered}
\sum_{t=1}^{N_{T}} P_{t, a}=\sum_{t=1}^{N_{T}} P_{t+\tau, a} \quad \forall a \in A^{t s} \\
\tau_{\text {min }, a}<\tau<\tau_{\text {max }, a} \quad \forall a \in A^{t s}
\end{gathered}
$$

where $\tau$ is the time shift of the appliance, $\tau_{\min , a}$ and $\tau_{\max , a}$ are the limits of the maximum allowable time shift and $A^{t s}$ is the set of all time shifting appliances. The optimization problem of the time shifting appliances needs to use the time shift $\tau$ as the decision variable rather than the power at each time instant.

In this work, PV systems have been considered as curtailable only, and their behavior has been considered inelastic to dynamic price signals. More information of the device level modeling can be found in [6].

\subsection{Price Adjustments}

Generally, day-ahead dynamic price for an end-user consists of three distinct parts: the day-ahead market price $\left(p_{t}^{D}\right)$, a flat network tariff $\left(p^{D S O}\right)$ and taxes $\left(p^{\operatorname{tax}}\right)$. Thus, the day-ahead price at any given time, $p_{t}$ can be expressed as,

$$
p_{t}=p_{t}^{D}+p^{D S O}+p^{t a x} .
$$

In the Netherlands, the day-ahead market price for a particular day has an hourly resolution and is available at 12:00 on the day before after the closure of the day-ahead market. The network tariff is usually fixed and is around EUR 150 per year. On top of these, the energy tax is applied based on the volume of energy consumption [5].

In this work, we aim to vary the flat network tariff to influence the end-users for shifting the flexible appliances from the peak hours to off-peak moments.

\subsubsection{Overloading Cost of the Transformer}

Overloading of a transformer occurs due to higher loads at connection points such as, for example, with charging a large number of EVs or the operation of domestic HPs. Thermal overloading generally affects the insulation of the transformer windings; however, the involved dynamics in the loading enable the transformer to be overloaded for some time. Consequently, the amount of flexibility that needs to be procured should be aligned with the thermal status and the respective cost of overloading of the transformer.

Based on the provided load-profiles and historical values of the transformer load, the TA generates a set of probable loading scenarios to tackle the inherent uncertainties. For each of these scenarios, it estimates the imminent loss-of-life and respective overloading cost of the transformers. To do so, the following steps are followed:

Step 1: In this step, the TA generates the set of scenarios for probable loading. This is to counter the uncertainties associated with real-time loading, deviations from day-ahead schedule and forecast errors of the local generation technologies. In reality, correlations exist between the loads of consecutive hours. Therefore, instead of calculating single values for each time step, pseudo-random profiles of loading are generated based on Copula theory [33].

A copula is a multivariate probability distribution where the marginal-distributions are uniform. According to the Sklar's theorem, if those marginal distributions are continuous, the copula is unique. Let $\mathrm{F}$ be a 24 -dimensional distribution function with continuous margins, $\left(F_{1}, F_{2} \ldots \ldots, F_{24}\right)$. A copula 
can be fitted, using the cumulative distribution functions (CDFs) of the historical load and the expected load profile provided by the aggregator. The marginal distribution here is the cumulative distribution functions generated by a Kernel Density Estimator (KDE). Then, the CDFs are used as inputs to generate the copula reflecting the correlation between the loads at different time steps. Next, the synthetic profiles generated from the fitted copula are transformed back to the original scale by applying the inverse cumulative distribution function. The whole process of scenario generation can be summarized by Figure 3.

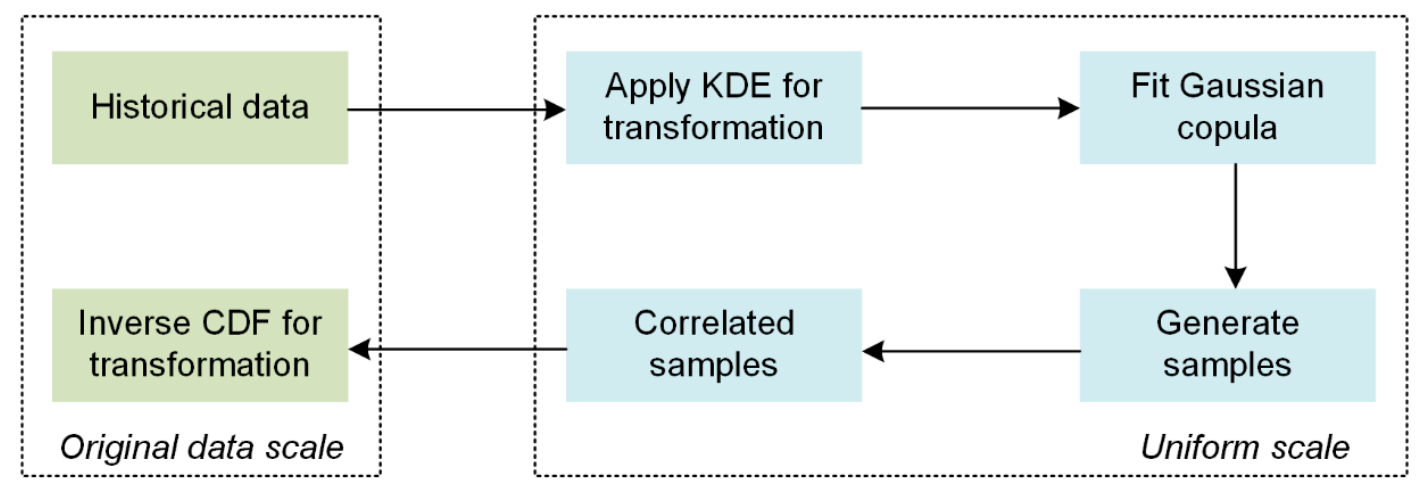

Figure 3. Generating day-ahead loading scenarios using copula.

Step 2: The expected load is converted to the resulting hottest-spot temperature, $\theta_{H}$, of the transformer. According to IEEE Std C57.12.00-1993, the hottest-spot temperature is defined as the highest temperature of the winding at the operating condition, and is the main element for calculating the expected life of a transformer [34]. To determine this, a ratio called the load multiplex, $K$, is calculated and then used to calculate the top oil temperature rise, $\Delta \theta_{T O}$, and the hottest-spot temperature rise, $\Delta \theta_{H}$, as:

$$
\begin{gathered}
K=\frac{\text { Expected load }}{\text { Rated load }} \\
\Delta \theta_{T O}=\Delta \theta_{T O, R}\left[\frac{K^{2} R+1}{R+1}\right] \\
\Delta \theta_{H}=\Delta \theta_{H, R} K^{2}
\end{gathered}
$$

where $\Delta \theta_{T O, R}$ is the top oil temperature rise at rated load, $\Delta \theta_{H, R}$ is hottest-spot temperature at the rated load and $R$ is the ratio of load loss at rated load to no-load loss at rated load. Then, $\theta_{H}$ is found by summing the ambient temperature $\theta_{A}$ with the above mentioned temperature rises.

$$
\theta_{H}=\theta_{A}+\Delta \theta_{T O}+\Delta \theta_{H}
$$

Step 3: In this step, the ageing acceleration factor $\left(F_{A A}\right)$ and equivalent ageing factor $\left(F_{\text {eqv }}\right)$ are calculated for the particular combination of loads and temperature for a duration of thirty min. This is due to the fact that degradation of the insulation is realized when the transformer is generally overloaded for half-an hour.

$$
\begin{aligned}
& F_{A A}=e^{\left[\frac{15000}{383}-\frac{15000}{\theta_{H}+273}\right]} \\
& F_{\text {eqv }}=\frac{\sum_{n=1}^{N} F_{A A} \Delta t_{n}}{\sum_{n=1}^{N} \Delta t_{n}}
\end{aligned}
$$

where $N$ is the number of time intervals, the duration of each is $\Delta t_{n}$ hours and normal insulation life of the transformer is $T_{\text {inl }}$ hours. According to IEEE standards, the normal insulation life of a well dried, oxygen free distribution transformer is $180,000 \mathrm{~h}$ or 20.55 years $[2,16]$. 
Step 4: Based on the calculated equivalent factor, per unit loss of life of the transformer $T_{l o l}$ is determined.

$$
T_{l o l}=\frac{F_{e q v} t}{T_{i n l}}
$$

The aging $\operatorname{cost} C_{a g}$ can be determined by the loss of life with the total owning cost (TOC) $C_{o}$ of the transformer. The TOC method is considered to be one of the most cost and resource efficient methods for economic analysis of a transformer [35]. In addition to the initial cost of the transformer, TOC considers the operation and maintenance cost of the transformer and is calculated over the life span of the asset. The TOC can be determined from purchase $\operatorname{cost} C_{P}$, cost of no-load loss $C_{N L}$ and cost of load loss $C_{L L}$ of the transformer [35].

$$
\begin{gathered}
C_{o}=C_{P}+C_{N L}+C_{L L} \\
C_{a g}=T_{l o l} C_{o}
\end{gathered}
$$

If $C_{a g}$ is greater than the aging cost at nominal rating of the transformer $C_{a g, R}$, then the overloading $\operatorname{cost} C_{O L}$ is determined from the arithmetic difference. Otherwise, the overloading cost is assumed to be zero.

$$
C^{O L}=\left\{\begin{array}{cc}
C_{a g}-C_{a g, R} & \text { when } C_{a g}>C_{a g, R} \\
0 & \text { otherwise }
\end{array}\right.
$$

\subsubsection{Calculation of Dynamic Tariff}

The TA calculates the dynamic tariff that satisfies the thermal constraints for each of the loading scenarios $s \in S$. This can be mathematically presented as the following optimization problem:

$$
\min \sum_{s=1, s \in S}^{s=s_{N}} \sum_{t=1, t \in T}^{t=t_{N}}\left(p_{t}^{D S O}-p^{D S O}\right) P_{t}^{s} \forall s \in S \text { if }\left(C_{t, s}^{O L}>0\right)
$$

subject to,

$$
\begin{gathered}
\overline{p_{t}^{D S O}}=\overline{p^{D S O}} \\
p_{t}^{D S O}=\frac{1}{5} \sum_{t_{f}=t-2}^{t+2} p_{t}^{D S O}+p_{a d j} \text { if } C_{t_{f}, S}^{O L}>0 \\
p_{a d j, t} \leq \max \left(C_{t, s}^{O L}\right) .
\end{gathered}
$$

$p_{t}^{D S O}$ is the decision variable and denotes the dynamic network tariff at each time step. $P_{t}^{s}$ is the load at time $t \in T$ in scenario, $s$. Constraints in Equation (21) dictate that the average network tariff should be the same for both normal operations and tariff-based DR cases. Constraints in Equations (22) and (23) limit the range of tariff adjustment in time and monetary values, respectively.

The resulting dynamic network tariff is sent to the Aggregator for updating the price for the end-users. This process is continued as an iterative process until the issues are resolved. In this work, we have limited the process to up to ten iterations. The whole process can be represented by the flowchart as shown in Figure 4. 


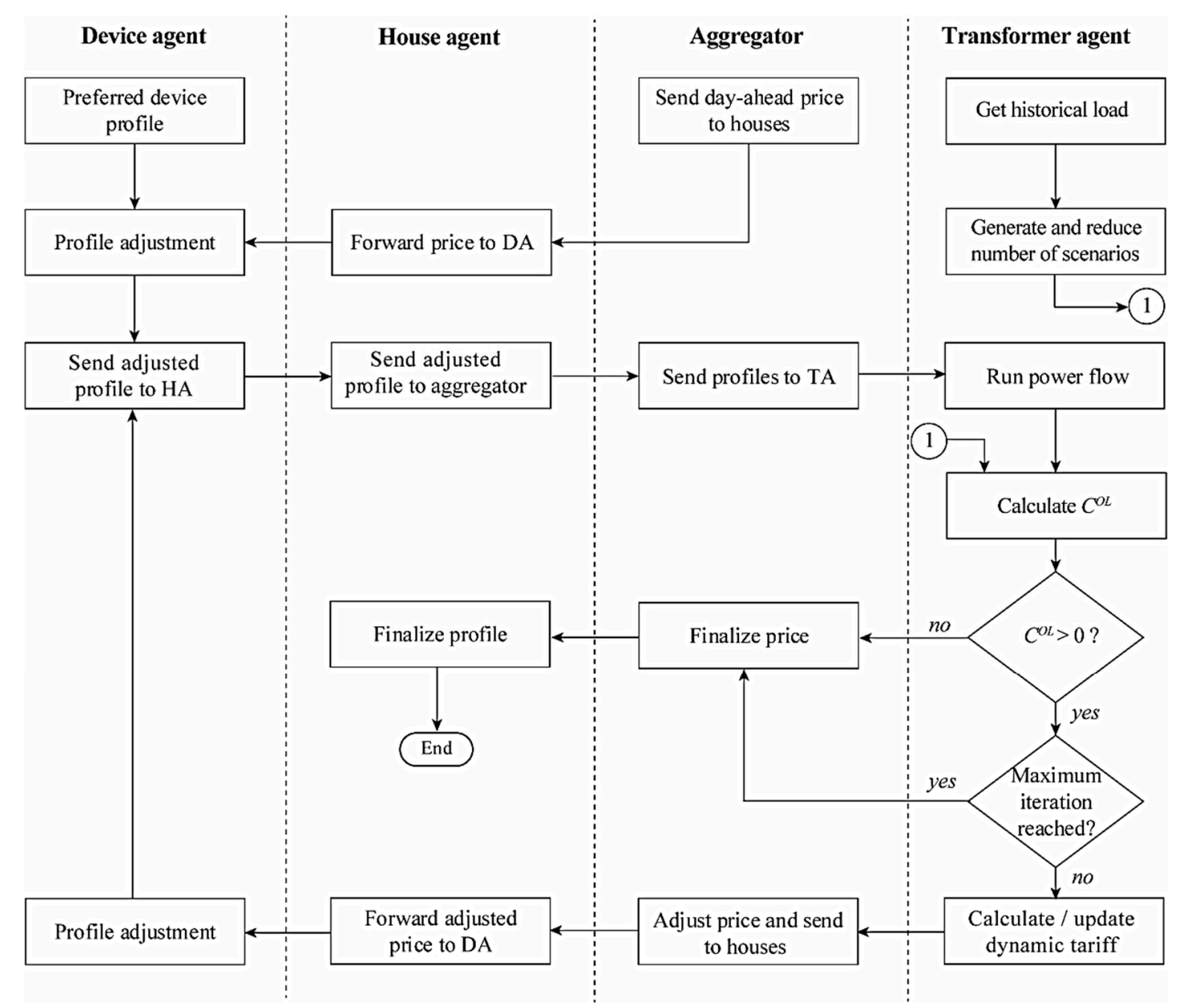

Figure 4. Flowchart depicting the processes.

\section{Simulation Setup}

\subsection{Load Modeling}

A bottom-up approach is adopted for the simulation test case. The residential loads are classified into seven categories: base load, EV, PV, heat pump, washing machine, dishwasher and refrigerator. The base load is considered to be comprised of the non-controllable devices. The required EV charge is modeled based on the driving distance, arrival and departure time [36]. The charging rate is assumed to range between 3 and $8 \mathrm{~kW}$. For the PV, the rated power varies between 2 and $5.5 \mathrm{~kW}$ per household. The heat pumps have a rated power of $0.7 \mathrm{~kW}$ to $2 \mathrm{~kW}$, with an additional $2 \mathrm{~kW}$ resistive heating element. The washing machine, dishwasher and refrigerator have a rated power in the range of $0.6-2 \mathrm{~kW}, 0.5-1.11 \mathrm{~kW}$ and $0.035-0.140 \mathrm{~kW}$, respectively. For more information about the loads, the reader is referred to [6]. A flat network tariff of EUR 0.06 is considered for $p^{D S O}$ [37]. The network tariff is adjusted in such a way so that the daily average is kept fixed at EUR 0.06 .

\subsection{Network}

A modified version of the IEEE European LV test network is used for the case study [38]. The network hosts 55 households with single phase connections. A $250 \mathrm{kVA}, 11 \mathrm{kV} / 0.416 \mathrm{kV}$ transformer supplies the LV segment from the MV bus. The resistance and reactance of the windings are $0.4 \%$ and $4 \%$ of the base values at the MV side, respectively. An additional LV feeder with an aggregated peak load of $100 \mathrm{~kW}$ is assumed at the substation. A constant power factor of 0.95 is used 
for the power flow calculation. Per unit values in the results are calculated considering the transformer rating as the base value.

\subsection{Simulation Platform}

The simulations have been performed with a time step of $15 \mathrm{~min}$. The MAS-based coordination is done in a MATLAB environment. Due to the relatively simpler form of the local optimization problems, the MATLAB Optimization Toolbox has been used to solve them. The power flow calculations are performed using the EPRI distribution system simulator, OpenDSS [39].

\section{Numerical Results}

In this section, simulation results are presented. First, the generated scenarios are briefly discussed, followed by a discussion on the effect of dynamic price on residential demand. Next, the impacts of the dynamic network tariff are explained, followed by the monthly performance of the proposed approach in terms of overall efficiency of managing the congestion.

\subsection{Scenarios of Loading}

Figure 5 shows the scenarios generated based on the historical loading data of the transformer and the scheduled demand for the following day based on the day-ahead dynamic price, as supplied by the aggregator. First, a set of 1000 scenarios are generated using Gaussian copula to represent the correlation among different time steps based on the data of previous one month. In order to simplify the calculation, this set of scenarios is reduced to 20 that closely represent the behavior of all the members of the set. Figure $5 \mathrm{c}$ shows that the set of reduced scenarios can capture the dynamics of all the time steps of the following day based on the day-ahead price. Thus, the set of reduced scenarios can address the uncertainties with the loading of the following day.
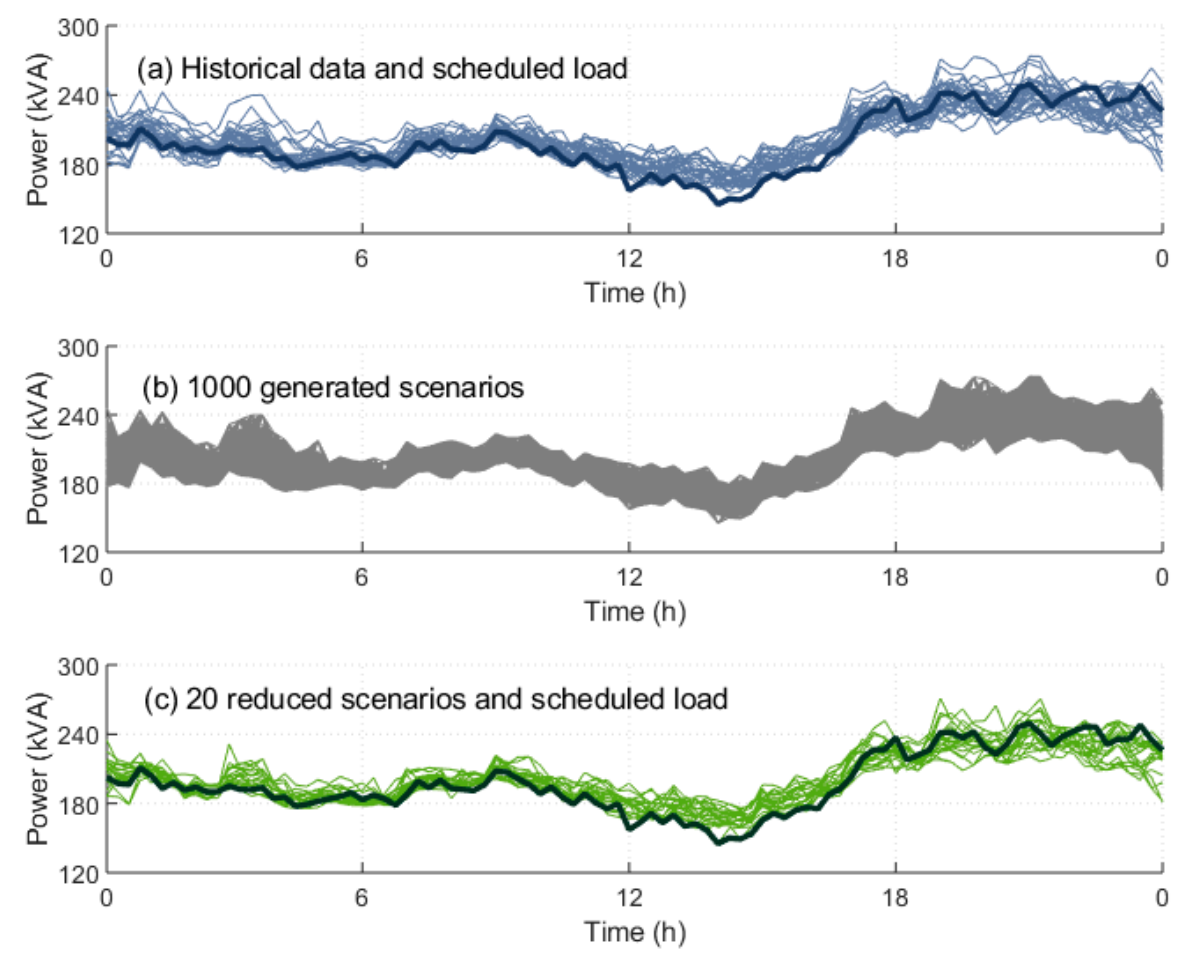

Figure 5. Transformer loading, (a) historical values of one month previous and based on scheduled loads for the following day, (b) generated scenarios based on Gaussian copula to represent the correlation and $(\mathrm{c})$ reduced scenarios and the scheduled load. 


\subsection{Effects of Dynamic Market Price}

Figure 6 illustrates the effects of the dynamic market price on the network load as a whole. The initial load profile represents the projected profiles of the households. The HAs adapt the initial profile based on the identical price from the aggregator. The price-adjusted profile shows distinct peaks when the price levels are lower. The HAs essentially minimize the total energy cost by optimizing the daily usage of all the appliances. To this end, the flexible appliances are mostly scheduled at times of lower price. Consequently, a reduced domestic energy cost is realized by the dynamic price levels at the expense of higher overall peak load.

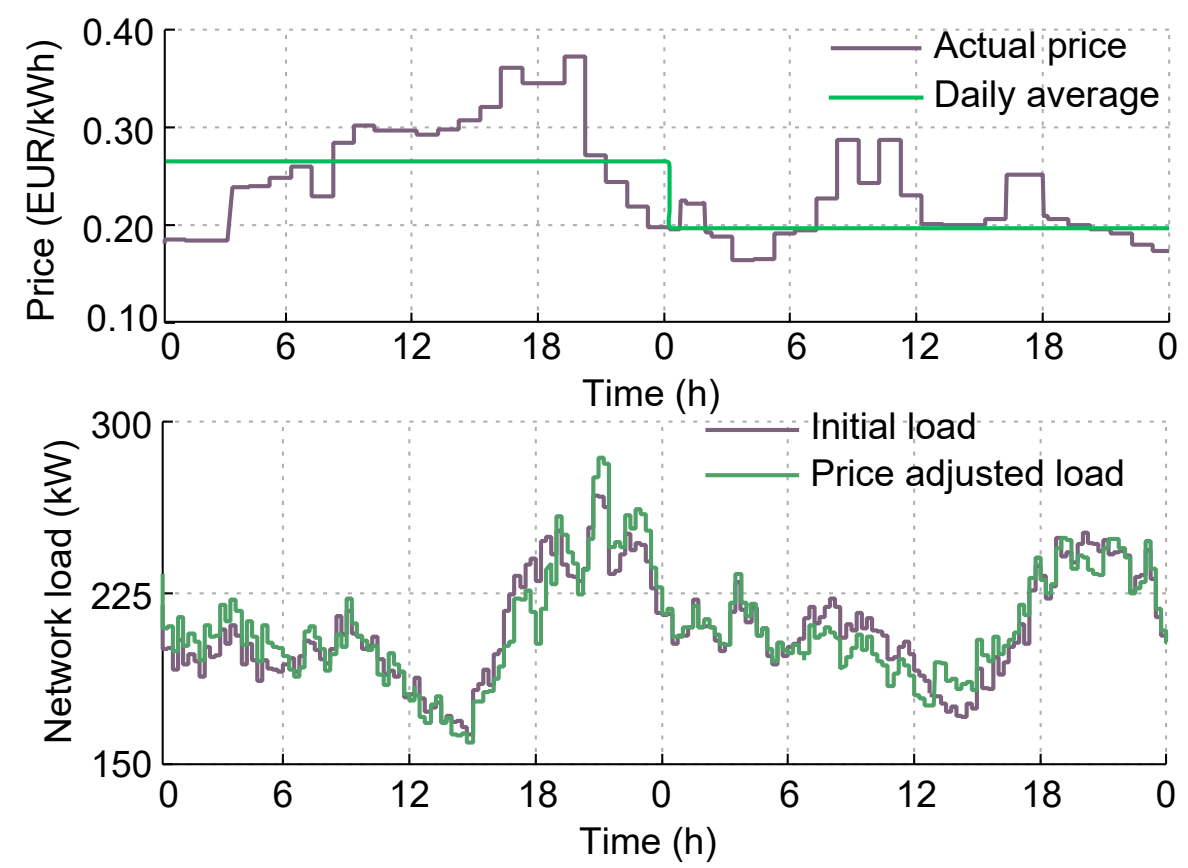

Figure 6. Effects of dynamic price on day-ahead scheduled load.

\subsection{Dynamic Network Tariff for Peak Reduction}

In order to resolve the congestion, the TA requests flexibility from the aggregator. This is done by introducing the variable network tariff, as discussed in Section 3.2. As the coordinator of the demand flexibility, the aggregator adjusts the price sent to the HAs by adding the variable tariff instead of the flat counterpart. Flexible appliances of the end-users react to this newly available price and try to shift loads from the peak moments. Figures 7 and 8 show the change in price and the resulting adjusted load profiles for two consecutive days, respectively. To make use of the decreasing price at the evening of the first day, EVs start charging almost simultaneously and, as a result, they cause congestion in the transformer. Similarly, the coincidence of the HP operations with the conventional evening peak leads to congestion on the second day.

Based on the overloading cost and expected loading scenarios, the TA changes the network tariff. The off-peak price level is further reduced due to lower tariff in order to maintain a constant daily average. The HAs respond to the new price levels by re-optimizing the flexible loads. The effect of the increase is reflected in the loads, as the peak load on the first day is lowered to 0.98 p.u. from 1.04 p.u. In both cases, the total energy consumption remains $4.95 \mathrm{MWh}$, since the flexible loads are merely shifted in time. 


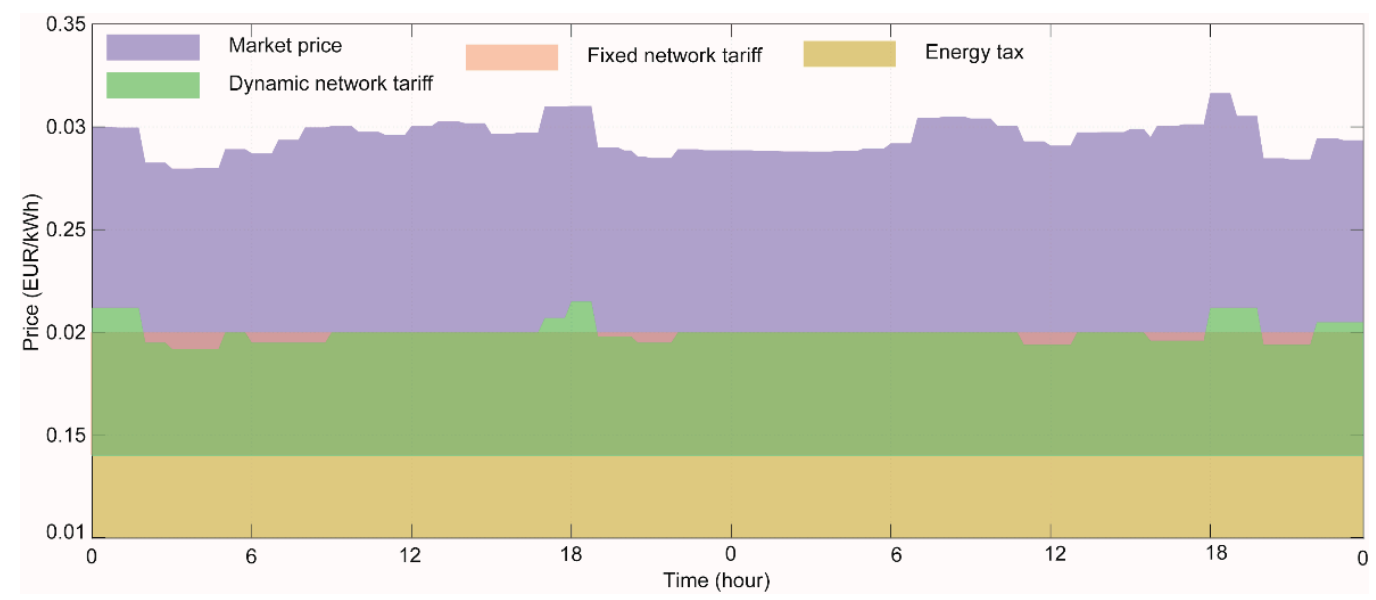

Figure 7. Electricity price breakdown in different cases.

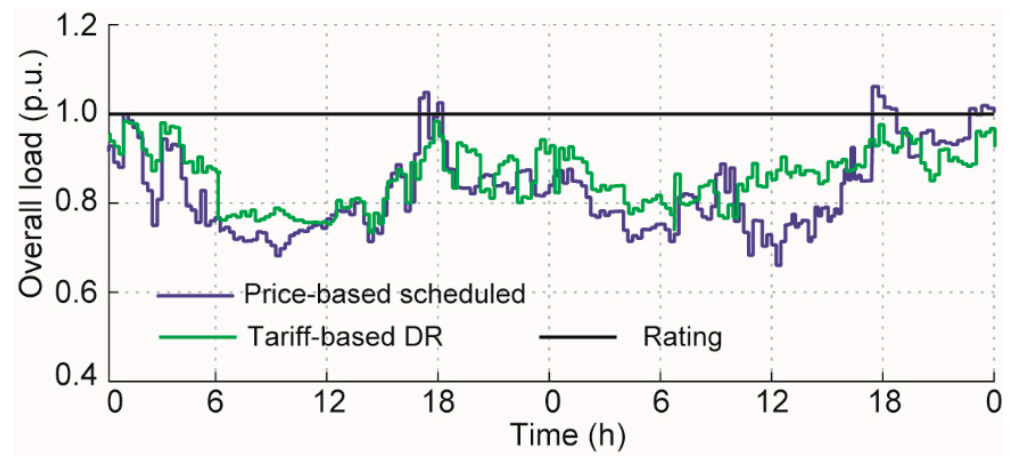

Figure 8. Simulated network load for two consecutive days.

\subsection{Monthly Assessment}

The overall monthly performance of the proposed approach is analyzed in terms of the congestion duration, network peak load and total supplied energy. This ensures the efficiency of the approach for resolving the congestion while maintaining the quality of supply. Table 1 depicts the comparative values of the uncontrolled load, the price-based scheduling and the proposed tariff-based DR scheme. The congestion duration is considerably reduced with the proposed approach at the expense of a drop in supplied energy. This happens due to the fact that some of the flexible appliances shift their energy consumption at later hours (possibly the next day) and, hence, were not considered within the simulation time window. The average monthly energy cost of the end-users has been reduced for the proposed method, although the objective of the proposed approach is to tackle imminent congestion in the network, and not to reduce the energy cost of the end-users. In reality, the end-users will not have to face the effect, since the daily average of the tariff will be the same. In other words, they will keep paying the same amount for the network tariff as before. The change in the cost is generated from the differences in day-ahead price at corresponding time steps.

Table 1. Comparative monthly assessment among different schemes.

\begin{tabular}{cccc}
\hline Performance Indicators & Uncontrolled Load & Price-Based Scheduling & Tariff-Based Dr \\
\hline Maximum load (p.u.) & 1.07 & 1.07 & 1.05 \\
Duration of congestion (h) & 11.50 & 9.75 & 1.75 \\
Supplied energy (MWh) & 148.22 & 148.34 & 148.07 \\
Average domestic cost (EUR) & 43.88 & 41.23 & 42.02 \\
\hline
\end{tabular}

The effects are also evident in the monthly load duration curve, as shown in Figure 9. The controlled case demonstrates a notable reduction in the peak demand and shifts the loads when the network tariff 
is lower. Since the total energy consumption is of similar order, the comfort levels of the end-users were also ensured.

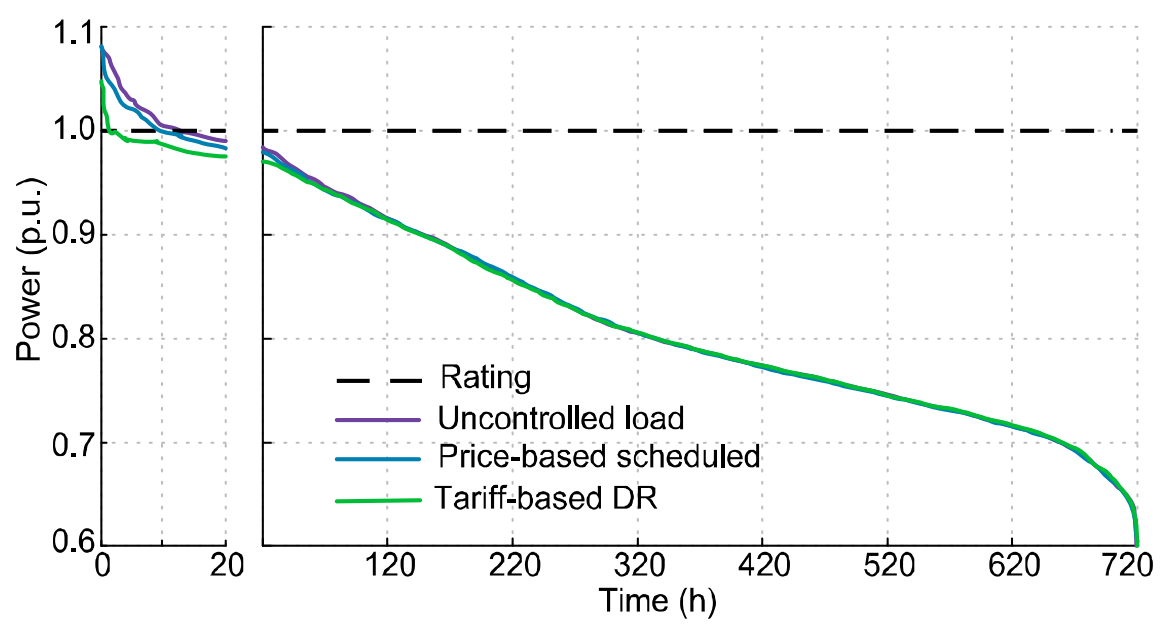

Figure 9. Monthly load duration curve.

\section{Conclusions}

A dynamic network tariff has been introduced in this paper that utilizes a hierarchical MAS-based architecture with distributed intelligence. The price-responsive behaviors of the loads have been modeled using a detailed bottom-up Markov Chain Monte Carlo approach. The loads are scheduled based on the dynamic prices available from the aggregator. If a case of network congestion is expected, the DSO adjusts the network tariff part of the price by varying it throughout the day. This is done on the basis of an estimated overloading cost resulting from the probable loading scenarios. The loading scenarios are generated using the Gaussian copula of the expected load from the aggregator and historical loading values of one month previous. The network tariff is raised around the peak hours while keeping the daily average fixed.

The proposed approach has been evaluated through simulations for 55 households in a modified form of the IEEE European LV test feeder. Simulation results reveal a notable relation between the dynamic price and congestion, which has been reported by a number of previous studies. The proposed approach appears to be efficient at managing congestions, as the total monthly congestion duration has been reduced up to $82 \%$. The monthly simulation has been performed on an Intel Core i7 computer with 8 GB of RAM. The proposed approach requires a simulation time of approximately $15 \mathrm{~min}$ compared to $12 \mathrm{~min}$ for the uncontrolled case.

Future research on this topic will be directed to efficient methods for local voltage control. The functionalities of the aggregator will be further updated with smarter learning techniques for more accurate price adjustments.

Author Contributions: Literature Research, N.H. and A.T.; Conceptualization, N.H.; Methodology, N.H.; Visualization, N.H. and A.T.; Writing original draft, N.H. and A.T.; Writing review and editing, N.H., P.N. and G.P. All authors have read and agreed to the published version of the manuscript.

Funding: This work is part of the research program ERA-net Smart Energy Systems with project number 651.001.012, which is financed by the Netherlands Organization for Scientific Research (NWO).

Conflicts of Interest: The authors declare no conflict of interest. 


\section{Nomenclature}

\section{List of Abbreviations}

\begin{tabular}{|c|c|c|c|}
\hline $\mathrm{CDF}$ & $\begin{array}{l}\text { Cumulative Distribution } \\
\text { Function }\end{array}$ & $\mathrm{KDE}$ & Kernel Density Estimator \\
\hline DA & Device Agent & LV & Low-voltage \\
\hline $\mathrm{DR}$ & Demand Response & MAS & Multi-Agent Systems \\
\hline DS & Dynamic Subsidy & MV & Medium-voltage \\
\hline DSO & Distribution System Operator & PV & Photovoltaics \\
\hline EPRI & Electric Power Research Institute & RES & Renewable Energy Sources \\
\hline EV & Electric Vehicle & TA & Transformer Agent \\
\hline HA & House Agent & TOC & Total Owning Cost \\
\hline $\mathrm{HP}$ & Heat Pump & & \\
\hline \multicolumn{4}{|c|}{ List of Symbols } \\
\hline \multicolumn{2}{|c|}{ Indices } & $\theta_{H}$ & hottest spot temperature \\
\hline$a$ & index of appliances & $\theta_{A}$ & ambient temperature \\
\hline$t$ & index of time steps & $F_{A A}$ & ageing acceleration factor \\
\hline$b f$ & index of buffer appliances & $F_{\text {eqv }}$ & equivalent factor \\
\hline ts & index of time shifting appliances & $C_{a g}$ & ageing cost \\
\hline$D$ & index of day & $T_{l o l}$ & loss of life \\
\hline$s$ & index of scenarios & $C^{O L}$ & overloading cost \\
\hline \multicolumn{2}{|l|}{ Sets } & \multicolumn{2}{|c|}{ Parameters } \\
\hline$A$ & set of appliances & $N_{t}$ & number of daily time steps \\
\hline$T$ & set of all time steps & $\Delta t$ & duration of a time step in hour \\
\hline$S$ & set of all scenarios & $x$ & buffer time \\
\hline \multicolumn{2}{|c|}{ Variables } & $\tau_{\min }$ & lower limit of allowable time shift \\
\hline$p$ & electricity price & $\tau_{\max }$ & upper limit of allowable time shift \\
\hline$P$ & active power & $\Delta \theta_{T O, R}$ & top oil temperature rise at rated load \\
\hline$k$ & time shift of buffer appliances & $\Delta \theta_{H, R}$ & hottest-spot temperature at rated load \\
\hline$\tau$ & $\begin{array}{l}\text { time shift of time shifting } \\
\text { appliances }\end{array}$ & $R$ & ratio of load loss to no-load loss \\
\hline K & load multiplex & $T_{i n l}$ & normal insulation life of transformer \\
\hline$\Delta \theta_{T O}$ & top oil temperature rise & $C_{P}$ & purchase cost \\
\hline$\Delta \theta_{H}$ & hottest spot temperature rise & $C_{N L}, C_{L L}$ & cost of no-load loss and load loss \\
\hline
\end{tabular}

\section{References}

1. Haque, A.N.M.M.; Nguyen, P.H.; Vo, T.H.; Bliek, F.W. Agent-based unified approach for thermal and voltage constraint management in LV distribution network. Electr. Power Syst. Res. 2017, 143, 462-473. [CrossRef]

2. Haque, A.N.M.M.; Shafiullah, D.S.; Nguyen, P.H.; Bliek, F.W. Real-Time Congestion Management in Active Distribution Network based on Dynamic Thermal Overloading Cost. In Proceedings of the Power Systems Computation Conference (PSCC), Genoa, Italy, 20-24 June 2016; pp. 1-7.

3. Veldman, E.; Verzijlbergh, R.A. Distribution Grid Impacts of Smart Electric Vehicle Charging from Different Perspectives. IEEE Trans. Smart Grid 2015, 6, 333-342. [CrossRef]

4. Haque, A.N.M.M. Smart Congestion Management in Active Distribution Networks; Eindhoven University of Technology: Eindhoven, The Netherlands, 2017.

5. Klaassen, E.A.M. Demand Response Benefits from a Power System Perspective; Eindhoven University of Technology: Eindhoven, The Netherlands, 2016.

6. Haque, A.; Nijhuis, M.; Ye, G.; Nguyen, P.; Bliek, F.; Slootweg, J. Integrating Direct and Indirect Load Control for Congestion Management in LV Networks. IEEE Trans. Smart Grid 2017, 10, 741-751. [CrossRef]

7. Li, Y.; Li, N. Mechanism design for reliability in demand response with uncertainty. In Proceedings of the American Control Conference, Seattle, WA, USA, 24-26 May 2017.

8. Ma, H.; Parkes, D.C.; Robu, V. Generalizing demand response through reward bidding. In Proceedings of the International Joint Conference on Autonomous Agents and Multiagent Systems AAMAS, São Paulo, Brazil, 8-12 May 2017. 
9. Hu, J.; You, S.; Lind, M.; Østergaard, J. Coordinated charging of electric vehicles for congestion prevention in the distribution grid. IEEE Trans. Smart Grid 2014, 5, 703-711. [CrossRef]

10. Liu, W.; Wu, Q.; Wen, F.; Ostergaard, J. Day-ahead congestion management in distribution systems through household demand response and distribution congestion prices. IEEE Trans. Smart Grid 2014, 5, 2739-2747. [CrossRef]

11. Nguyen, P.H.; Kling, W.L.; Ribeiro, P.F. A game theory strategy to integrate distributed agent-based functions in smart grids. IEEE Trans. Smart Grid 2013, 4, 568-576. [CrossRef]

12. Palensky, P.; Dietrich, D. Demand side management: Demand response, intelligent energy systems, and smart loads. IEEE Trans. Ind. Inf. 2011, 7, 381-388. [CrossRef]

13. Ghazvini, M.A.F.; Lipari, G.; Pau, M.; Ponci, F.; Monti, A.; Soares, J.; Castro, R.; Vale, Z. Congestion management in active distribution networks through demand response implementation. Sustain. Energy Grids Netw. 2019, 17, 100185. [CrossRef]

14. Garcia, T.S.; Shafie-Khah, M.; Osório, G.J.; Catalão, J.P.S. Optimal bidding strategy of responsive demands in a new decentralized market-based scheme. In Proceedings of the 17th IEEE International Conference on Environment and Electrical Engineering and 1st IEEE Industrial and Commercial Power Systems Europe, Milan, Italy, 6-9 June 2017.

15. Chai, B.; Chen, J.; Yang, Z.; Zhang, Y. Demand response management with multiple utility companies: A two-level game approach. IEEE Trans. Smart Grid 2014, 5, 722-731. [CrossRef]

16. Haque, A.N.M.M.; Nguyen, P.H.; Bliek, F.W.; Slootweg, J.G. Demand response for real-time congestion management incorporating dynamic thermal overloading cost. Sustain. Energy Grids Netw. 2017, 10, 65-74. [CrossRef]

17. Nizami, M.S.H.; Haque, A.N.M.M.; Nguyen, P.H.; Bliek, F.W. HEMS as Network Support Tool: Facilitating Network Operator in Congestion Management and Overvoltage Mitigation. In Proceedings of the 16th International Conference on Environment and Electrical Engineering (EEEIC), Florence, Italy, 6-8 June 2016; pp. 1-6.

18. Torbaghan, S.S.; Gibescu, M.; Rawn, B.G.; van der Meijden, M. A market-based transmission planning for HVDC grid-Case study of the North Sea. IEEE Trans. Power Syst. 2015, 30, 784-794. [CrossRef]

19. Hu, J.; Saleem, A.; You, S.; Nordström, L.; Lind, M.; Østergaard, J. A multi-agent system for distribution grid congestion management with electric vehicles. Eng. Appl. Artif. Intell. 2015, 38, 45-58. [CrossRef]

20. Zhang, C.; Ding, Y.; Østergaard, J.; Bindner, H.W.; Nordentoft, N.C.; Hansen, L.H.; Brath, P.; Cajar, P.D. A flex-market design for flexibility services through DERs. In Proceedings of the 4th IEEE/PES Innovative Smart Grid Technologies Europe, Copenhagen, Denmark, 6-9 October 2013.

21. Huang, S.; Wu, Q.; Cheng, L.; Liu, Z.; Zhao, H. Uncertainty Management of Dynamic Tariff Method for Congestion Management in Distribution Networks. IEEE Trans. Power Syst. 2016, 3053, 1-12. [CrossRef]

22. Huang, S.; Wu, Q. Dynamic Subsidy Method for Congestion Management in Distribution Networks. IEEE Trans. Smart Grid 2018, 9, 2140-2151. [CrossRef]

23. Zhao, J.; Wang, Y.; Song, G.; Li, P.; Wang, C.; Wu, J. Congestion Management Method of Low-Voltage Active Distribution Networks Based on Distribution Locational Marginal Price. IEEE Access 2019, 7, 32240-32255. [CrossRef]

24. Lampropoulos, I.; van den Broek, M.; van der Hoofd, E.; Hommes, K.; van Sark, W. A system perspective to the deployment of flexibility through aggregator companies in the Netherlands. Energy Policy 2018, 118, 534-551. [CrossRef]

25. Biggar, D.; Reeves, A. Network Pricing for the Prosumer Future: Demand-Based Tariffs or Locational Marginal Pricing? In Future of Utilities-Utilities of the Future: How Technological Innovations in Distributed Energy Resources Will Reshape the Electric Power Sector; Academic Press: Cambridge, MA, USA, 2016.

26. Torbaghan, S.S.; Blaauwbroek, N.; Nguyen, P.; Gibescu, M. Local market framework for exploiting flexibility from the end users. In Proceedings of the 2016 13th International Conference on the European Energy Market (EEM), Porto, Portugal, 6-9 June 2016; pp. 1-6. [CrossRef]

27. Eid, C.; Codani, P.; Perez, Y.; Reneses, J.; Hakvoort, R. Managing electric flexibility from Distributed Energy Resources: A review for incentives, aggregation and market design. Renew. Sustain. Energy Rev. 2015, 64, 237-247. [CrossRef]

28. Nwulu, N.I.; Xia, X. Optimal dispatch for a microgrid incorporating renewables and demand response. Renew. Energy 2017, 101, 16-28. [CrossRef] 
29. Pratt, B.A.; Krishnamurthy, D.; Ruth, M. Transactive Home Energy Management Systems. IEEE Electrif. Mag. 2016, 4, 8-14. [CrossRef]

30. Minniti, S.; Haque, N.; Nguyen, P.; Pemen, G. Local markets for flexibility trading: Key stages and enablers. Energies 2018, 11, 3074. [CrossRef]

31. Nijhuis, M.; Gibescu, M.; Cobben, J.F.G. Bottom-up Markov Chain Monte Carlo approach for scenario based residential load modelling with publicly available data. Energy Build. 2016, 112, 121-129. [CrossRef]

32. Flexible Power Alliance Interface (FPAI). Available online: http://www.flexiblepower.org/downloads/ (accessed on 21 December 2019).

33. Ni, F.; Nguyen, P.H.; Cobben, J.F.G. Basis-Adaptive Sparse Polynomial Chaos Expansion for Probabilistic Power Flow. IEEE Trans. Power Syst. 2017, 32, 694-704. [CrossRef]

34. ABB. Distribution Transformer Handbook IEC/CENELEC Related Specifications ABB; ABB: Zurich, Switzerland, 2003.

35. Downing, D.J.; McConnell, B.W.; Barnes, P.R.; Hadley, S.W.; van Dyke, J.W. Economic Analysis of Efficient Distribution Transformer Trends; Oak Ridge National Laboratory: Oak Ridge, TN, USA, 1998.

36. Verzijlbergh, R.A.; Lukszo, Z.; Veldman, E.; Slootweg, J.G.; Ilic, M. Deriving electric vehicle charge profiles from driving statistics. In Proceedings of the IEEE Power \& Energy Society General Meeting, Detroit, MI, USA, 24-28 July 2011; pp. 1-6.

37. Verzijlbergh, R.A. The Power of Electric Vehicles; Delft University of Technology: Delft, The Netherlands, 2013.

38. IEEE PES Distribution Test Feeder Working Group. Distribution Test Feeders. Available online: https: //site.ieee.org/pes-testfeeders/resources/ (accessed on 21 December 2019).

39. EPRI. OpenDSS, Distribution System Simulator. Available online: http://sourceforge.net/projects/electricdss (accessed on 26 June 2018).

(C) 2020 by the authors. Licensee MDPI, Basel, Switzerland. This article is an open access article distributed under the terms and conditions of the Creative Commons Attribution (CC BY) license (http://creativecommons.org/licenses/by/4.0/). 